\title{
Hadron Spectrum in a Two-Colour Baryon-Rich Medium
}

\author{
Simon Hands ${ }^{a, b}$, Peter Sitch $^{a}$, and Jon-Ivar Skullerud ${ }^{c, d}$ \\ ${ }^{a}$ Department of Physics, Swansea University, \\ Singleton Park, Swansea SA2 8PP, U.K.1 \\ ${ }^{b}$ Isaac Newton Institute for Mathematical Sciences, \\ 20 Clarkson Road, Cambridge CB3 OEH, U.K. \\ ${ }^{c}$ School of Mathematics, Trinity College, Dublin 2, Ireland. \\ ${ }^{d}$ Department of Mathematical Physics, National University of Ireland, \\ Maynooth, County Kildare, Ireland 2
}

\begin{abstract}
The hadron spectrum of SU(2) lattice gauge theory with two flavours of Wilson quark is studied on an $8^{3} \times 16$ lattice using all-to-all propagators, with particular emphasis on the dependence on quark chemical potential $\mu$. As $\mu$ is increased from zero the diquark states with non-zero baryon number $B$ respond as expected, while states with $B=0$ remain unaffected until the onset of non-zero baryon density at $\mu=m_{\pi} / 2$. Post onset the pi-meson mass increases in accordance with chiral perturbation theory while the rho becomes lighter. In the diquark sector a Goldstone state associated with a superfluid ground state can be identified. A further consequence of superfluidity is an approximate degeneracy between mesons and baryons with the same spacetime and isospin quantum numbers. Finally we find tentative evidence for the binding of states with kaon quantum numbers within the baryonic medium.
\end{abstract}

PACS: 11.15.Ha, 12.40.Yx, 21.65.+f, 24.85.+p

Keywords: chemical potential, hadron spectrum, nuclear matter

\section{Introduction}

At large baryon chemical potential $\mu_{B}$ the properties of QCD are expected to change as the system moves from a confined nuclear matter phase to a deconfined quark matter phase where the relevant degrees of freedom are quarks and gluons. At low temperature $T$ and high $\mu_{B}$, the attraction between quarks is believed to be sufficient to promote diquark Cooper pairing leading to a colour superconducting ground state. Weak-coupling techniques can be used at asymptotic densities and have revealed a superconducting phase known as the colour-flavour locked phase. However as density is reduced towards

\footnotetext{
${ }^{1}$ Permanent address

${ }^{2}$ Permanent address
} 
phenomenologically reasonable values, the precise nature of the ground state appears very sensitive both to additional parameters such as isospin chemical potential and strange quark mass, and also to the nature of the non-perturbative assumptions made in the calculation. It seems natural to use Lattice QCD to investigate these issues, but unfortunately whilst the lattice has been used very successfully to investigate QCD with $T>0$, the well known "Sign Problem" has made progress for $\mu_{B} / T \gg 1$ impossible.

Orthodox simulation techniques can be applied, however, to the case of two colour QCD $\left(\mathrm{QC}_{2} \mathrm{D}\right)$ with gauge group $\mathrm{SU}(2)$. Whilst this theory differs in important ways from QCD, for instance in having bosonic baryons in the spectrum, and in having a superfluid, rather than superconducting, ground state at large $\mu_{B}$, it remains the simplest gauge theory in which a systematic non-perturbative treatment of a baryonic medium is possible. Moreover, recent simulations [1] have provided evidence that there exist two distinct forms of two color matter: the dilute Bose gas formed from diquark bound states which forms at onset, ie. for $\mu_{B}>\mu_{B o}=M_{\pi}$, in which superfluidity arises via BoseEinstein condensation of scalar diquarks, is supplanted at larger densities by a deconfined "quark matter" phase in which a system of degenerate quarks is disrupted by BCS condensation at the Fermi surface. Studies in this regime may have qualitative or even quantitative relevance for QCD quark matter, particularly in the non-Goldstone sector. For instance, Schäfer [2] has stressed how the impact of instantons on the excitation spectrum at high baryon density could be elucidated by lattice simulations.

In this Letter we study the $\mu_{B}$-dependence of the hadron spectrum in both meson and baryon sectors of $\mathrm{QC}_{2} \mathrm{D}$ with $N_{f}=2$ flavours of Wilson quark, which we find to vary dramatically as the onset from vacuum to a ground state with non-zero baryon density is traversed. We build on the pioneering work of the Hiroshima group [3], who found that beyond onset the pion mass did not change noticeably, but the rho meson became significantly lighter, so that the level-ordering is reversed. Two baryon channels were also studied but no significant $\mu_{B}$-dependence found. This work was performed on small lattices, and only looked at a few states. One of our aims is to improve and update their results. We also study the nature of the Goldstone mode associated with superfluidity, as done for $\mathrm{QC}_{2} \mathrm{D}$ with staggered lattice fermions in [4], and expose the specifically two colour phenomenon of "meson-baryon" mixing in the superfluid state, whereby since $B$ is no longer conserved, states interpolated by mesonic operators $q \bar{q}$ and diquark operators $q q$ have identical quantum numbers and hence exhibit an approximate degeneracy. Finally, we make the first measurements of strange meson masses in a baryonic medium, and find tentative evidence for bound states of kaons in nuclei.

\section{Formulation}

The gauge-invariant lattice action with $N_{f}=2$ degenerate fermion flavours is [1]

$$
S=\bar{\psi}_{1} M(\mu) \psi_{1}+\bar{\psi}_{2} M(\mu) \psi_{2}-\kappa j\left(\bar{\psi}_{1} K \bar{\psi}_{2}^{T}-\psi_{2}^{T} K \psi_{1}\right)
$$


with $M$ the conventional Wilson fermion matrix (with lattice spacing $a=1$ )

$$
M_{x y}(\mu)=\delta_{x y}-\kappa \sum_{\nu}\left[\left(1-\gamma_{\nu}\right) e^{\mu \delta_{\nu 0}} U_{\nu}(x) \delta_{y, x+\hat{\nu}}+\left(1+\gamma_{\nu}\right) e^{-\mu \delta_{\nu 0}} U_{\nu}^{\dagger}(y) \delta_{y, x-\hat{\nu}}\right]
$$

$\kappa$ the hopping parameter, $\mu$ the quark chemical potential, and $j$ the strength of an $\mathrm{SU}(2)_{L} \otimes \mathrm{SU}(2)_{R}$-invariant diquark source term needed to regularise IR fluctuations in the superfluid phase, which should be extrapolated to zero to reach the physical limit. The subscript on the fermion fields is a flavour index. The anti-unitary operator $K=$ $K^{T} \equiv C \gamma_{5} \tau_{2}$, where $C \gamma_{\mu} C^{-1}=-\gamma_{\mu}^{T}=-\gamma_{\mu}^{*}$ and the Pauli matrix $\tau_{2}$ acts on colour indices. A useful relation is

$$
M^{T}(\mu)=-K \gamma_{5} M(-\mu) K \gamma_{5}
$$

The hadronic states examined in this paper are $q \bar{q}$ mesons and $q q, \bar{q} \bar{q}$ diquark baryons and anti-baryons. In all cases we use local interpolating operators of the form $\bar{\psi}(x) \Gamma \psi(x)$, $\psi^{T}(x) K \Gamma \psi(x), \bar{\psi}(x) K \bar{\Gamma} \bar{\psi}^{T}(x)$. The matrix $\Gamma=\gamma_{0} \bar{\Gamma}^{\dagger} \gamma_{0}$ determines the spacetime quantum numbers of the hadron, with inclusion of the $K$ factor ensuring that mesons and baryons with the same $\Gamma$ have the same $J^{P}$. In this letter we will focus on states with $\Gamma \in\left\{\mathbb{1}, \gamma_{5}, \gamma_{j}, i \gamma_{5} \gamma_{j}\right\}$ with $j=1, \ldots, 3$ corresponding to $J^{P} \in\left\{0^{+}, 0^{-}, 1^{-}, 1^{+}\right\}$.

\subsection{Fermion Propagators}

The fermion action (1) can be written in the form $\bar{\Psi} \mathcal{M}(\mu, j) \Psi$ where $\bar{\Psi} \equiv\left(\bar{\psi}_{1}, \psi_{2}^{T}, \bar{\psi}_{2}, \psi_{1}^{T}\right)$ and $\Psi \equiv\left(\psi_{1}, \bar{\psi}_{2}^{T}, \psi_{2}, \bar{\psi}_{1}^{T}\right)^{T}$. It has the form:

$$
\mathcal{M}=\left(\begin{array}{cc}
A & 0 \\
0 & \bar{A}
\end{array}\right) \quad \text { with } \quad A=\frac{1}{2}\left(\begin{array}{cc}
M & -\kappa j K \\
\kappa j K & -M^{T}
\end{array}\right)
$$

and $\bar{A}(j)=A(-j)$. Now consider the propagator

$$
\langle\Psi(x) \bar{\Psi}(y)\rangle \equiv\left(\begin{array}{cccc}
S_{11} & S_{12} & 0 & 0 \\
\bar{S}_{21} & \bar{S}_{22} & 0 & 0 \\
0 & 0 & S_{22} & S_{21} \\
0 & 0 & \bar{S}_{12} & \bar{S}_{11}
\end{array}\right)
$$

where the zero entries arise from the assumption of isospin symmetry. This symmetry also implies that

$$
S_{22}=S_{11} \equiv S_{N} ; \quad \bar{S}_{11}=\bar{S}_{22} \equiv \bar{S}_{N} ; \quad S_{21}=-S_{12} \equiv S_{A} ; \quad \bar{S}_{12}=-\bar{S}_{21} \equiv-\bar{S}_{A},
$$

where the subscripts denote "normal" and "anomalous" propagation. Anomalous propagation arises from particle-hole mixing in a superfluid ground state; on a finite volume $S_{A}$ vanishes in the limit $j \rightarrow 0$. 


\subsection{Mesons}

The isovector $(I=1)$ meson operators $M^{1}$ are given by $\bar{\psi}_{1} \Gamma \psi_{2}, \bar{\psi}_{2} \Gamma \psi_{1}$ and $\left(\bar{\psi}_{1} \Gamma \psi_{1}-\right.$ $\left.\bar{\psi}_{2} \Gamma \psi_{2}\right) / \sqrt{ } 2$. The charged meson correlator is then

$$
\begin{aligned}
\left\langle M^{1}(x) M^{1 \dagger}(y)\right\rangle & =\left\langle\bar{\psi}_{1}(x) \Gamma \psi_{2}(x) \bar{\psi}_{2}(y) \bar{\Gamma} \psi_{1}(y)\right\rangle \\
& =-\operatorname{Tr}\left[S_{N}(y, x) \Gamma S_{N}(x, y) \bar{\Gamma}\right]+\operatorname{Tr}\left[\bar{S}_{A}(y, x) \Gamma S_{A}(x, y) \bar{\Gamma}^{T}\right]
\end{aligned}
$$

For the neutral meson correlator, the "disconnected" parts made up from the product of two traces cancel because of isospin symmetry. The connected parts are:

$$
\begin{aligned}
\left\langle M^{1}(x) M^{1 \dagger}(y)\right\rangle= & \left\langle\bar{\psi}_{1}(x) \Gamma \psi_{1}(x) \bar{\psi}_{1}(y) \bar{\Gamma} \psi_{1}(y)\right\rangle_{c}-\left\langle\bar{\psi}_{1}(x) \Gamma \psi_{1}(x) \bar{\psi}_{2}(y) \bar{\Gamma} \psi_{2}(y)\right\rangle_{c} \\
& -\left\langle\bar{\psi}_{2}(x) \Gamma \psi_{2}(x) \bar{\psi}_{1}(y) \bar{\Gamma} \psi_{1}(y)\right\rangle_{c}+\left\langle\bar{\psi}_{2}(x) \Gamma \psi_{2}(x) \bar{\psi}_{2}(y) \bar{\Gamma} \psi_{2}(y)\right\rangle_{c} \\
= & -\operatorname{Tr}\left[S_{N}(y, x) \Gamma S_{N}(x, y) \bar{\Gamma}\right]+\operatorname{Tr}\left[\bar{S}_{A}(y, x) \Gamma S_{A}(x, y) \bar{\Gamma}^{T}\right]
\end{aligned}
$$

and so the degeneracy between neutral and charged isovector mesons is manifest. The isoscalar $(I=0)$ meson $M^{0}=\left(\bar{\psi}_{1} \Gamma \psi_{1}+\bar{\psi}_{2} \Gamma \psi_{2}\right) / \sqrt{ } 2$ has the correlator

$$
\begin{aligned}
\left\langle M^{0}(x) M^{0 \dagger}(y)\right\rangle= & 2 \operatorname{Tr}\left[S_{N}(x, x) \Gamma\right] \operatorname{Tr}\left[S_{N}(y, y) \bar{\Gamma}\right] \\
& -\operatorname{Tr}\left[S_{N}(y, x) \Gamma S_{N}(x, y) \bar{\Gamma}\right]-\operatorname{Tr}\left[\bar{S}_{A}(y, x) \Gamma S_{A}(x, y) \bar{\Gamma}^{T}\right] .
\end{aligned}
$$

In this case there is a disconnected term, and the anomalous term has the opposite sign to that of the isovector meson. From now on the isovector $0^{-}$and $1^{-}$mesons will be referred to as the pion and rho respectively. Note that for $j=\mu=0$ the difference between $I=0$ and $I=1$ is entirely due to disconnected diagrams, which must therefore account for the $\pi-\eta^{\prime}$ mass splitting. For $\mu, j \neq 0$ this need not be the case.

In this Letter we for the first time use lattice techniques to study the spectrum of kaons in a dense baryonic medium. We model the kaon states by isovector mesons (7) in which one of the quark flavours does not feel the chemical potential (for details of how this is achieved see Sec. 3). The resulting propagator thus approximates the behaviour of an $s$ quark in a medium made up from $u$ and $d$ quarks, although at this exploratory stage we make no attempt to give the strange quarks a realistic mass but simply assume $\kappa_{s}=\kappa$. The states studied here correspond to $K^{ \pm}\left(J^{P}=0^{-}\right)$and $K^{* \pm}\left(J^{P}=1^{-}\right)$.

\subsection{Diquarks}

An isoscalar diquark is given by

$$
D^{0}(x)=\frac{1}{\sqrt{ } 2}\left(\psi_{1}^{T}(x) K \bar{\Gamma} \psi_{2}(x)-\psi_{2}^{T}(x) K \bar{\Gamma} \psi_{1}(x)\right)
$$

implying a correlator:

$$
\begin{aligned}
2\left\langle D^{0 \dagger}(x) D^{0}(y)\right\rangle & =\left\langle\bar{\psi}_{1}(x) \Gamma K \bar{\psi}_{2}^{T}(x) \psi_{1}^{T}(y) K \bar{\Gamma} \psi_{2}(y)\right\rangle-\left\langle\bar{\psi}_{1}(x) \Gamma K \bar{\psi}_{2}^{T}(x) \psi_{2}^{T}(y) K \bar{\Gamma} \psi_{1}(y)\right\rangle \\
& -\left\langle\bar{\psi}_{2}(x) \Gamma K \bar{\psi}_{1}^{T}(x) \psi_{1}^{T}(y) K \bar{\Gamma} \psi_{2}(y)\right\rangle+\left\langle\bar{\psi}_{2}(x) \Gamma K \bar{\psi}_{1}^{T}(x) \psi_{2}^{T}(y) K \bar{\Gamma} \psi_{1}(y)\right\rangle \\
& =4 \operatorname{Tr}\left[\bar{S}_{A}(x, x) \Gamma K\right] \operatorname{Tr}\left[S_{A}(y, y) K \bar{\Gamma}\right] \\
& +2 \operatorname{Tr}\left[S_{N}(y, x) \Gamma K \bar{S}_{N}(x, y) \bar{\Gamma}^{T} K\right]+2 \operatorname{Tr}\left[S_{N}(y, x) \Gamma K \bar{S}_{N}(x, y) K \bar{\Gamma}\right] .
\end{aligned}
$$


The last two terms arising from connected diagrams cancel if $K \bar{\Gamma}^{T} K=\bar{\Gamma}$, which is the case for $\Gamma=i \gamma_{5} \gamma_{j}$, and add up if $K \bar{\Gamma}^{T} K=-\bar{\Gamma}$ implying $\Gamma \in\left\{\mathbb{1}, \gamma_{5}, \gamma_{j}\right\}$. Thus the only baryon states available in the $I=0$ sector are $0^{+}, 0^{-}$and $1^{-}$; this is just the Pauli exclusion principle in action. The isovector diquark correlator is

$$
\left\langle D^{1 \dagger}(x) D^{1}(y)\right\rangle=\operatorname{Tr}\left[S_{N}(y, x) \Gamma K \bar{S}_{N}(x, y) \bar{\Gamma}^{T} K\right]-\operatorname{Tr}\left[S_{N}(y, x) \Gamma K \bar{S}_{N}(x, y) K \bar{\Gamma}\right] .
$$

Here the disconnected diagrams cancel, and the connected terms only add up for $\Gamma=$ $i \gamma_{5} \gamma_{j}$ implying $J^{P}=1^{+}$.

Spontaneous symmetry breaking of the $\mathrm{U}(1)_{B}$ symmetry $\psi \mapsto e^{i \alpha} \psi, \bar{\psi} \mapsto \bar{\psi} e^{-i \alpha}$ in the superfluid phase (the symmetry is explicitly broken when $j \neq 0$ ) can be probed by studying two special diquark states in the isoscalar $0^{+}$sector which we refer to as "Higgs" and "Goldstone" [5]. The operators, which by construction yield correlation functions symmetric under Euclidean time reversal, are given by

$$
D^{ \pm}(x)=\bar{\psi}_{1}(x) K \bar{\psi}_{2}^{T}(x) \pm \psi_{1}^{T}(x) K \psi_{2}(x),
$$

where the Higgs is ' + ' and the Goldstone ' - '. After Wick contraction the correlators are

$$
\begin{aligned}
\left\langle D^{ \pm}(x) D^{ \pm}(y)\right\rangle & =\operatorname{Tr}\left[K \bar{S}_{A}(x, x)\right] \operatorname{Tr}\left[K \bar{S}_{A}(y, y)\right]+\operatorname{Tr}\left[K S_{A}(x, x)\right] \operatorname{Tr}\left[K S_{A}(y, y)\right] \\
& \left. \pm\left(\operatorname{Tr}\left[K \bar{S}_{A}(x, x)\right] \operatorname{Tr}\left[K S_{A}(y, y) r\right]\right)+\operatorname{Tr}\left[K S_{A}(x, x)\right] \operatorname{Tr}\left[K \bar{S}_{A}(y, y)\right]\right) \\
& -\left(\operatorname{Tr}\left[\bar{S}_{A}(y, x) K \bar{S}_{A}(x, y) K\right]+\operatorname{Tr}\left[S_{A}(y, x) K S_{A}(x, y) K\right]\right) \\
& \pm\left(\operatorname{Tr}\left[S_{N}(y, x) K \bar{S}_{N}(x, y) K\right]+\operatorname{Tr}\left[\bar{S}_{N}(y, x) K S_{N}(x, y) K\right]\right) .
\end{aligned}
$$

With the choice of diquark source specified in (1), in the superfluid phase the $\mathrm{U}(1)_{B}$ symmetry is preferentially broken by a condensate $\left\langle D^{+}\right\rangle \neq 0$, and as the name implies, in the $j \rightarrow 0$ limit a massless Goldstone mode is interpolated by $D^{-}$. The degeneracy between Higgs and Goldstone is principally broken by connected diagrams formed from normal propagators.

\section{Numerical Method}

We studied an ensemble of gauge configurations generated on a $8^{3} \times 16$ lattice at various values of $\mu$ using a Hybrid Monte Carlo algorithm with the fermion action (1) supplemented by a standard Wilson gauge action [1]. The parameters were $\beta=1.7, \kappa=0.178$; studies of the string tension suggest a "physical" lattice spacing $a=0.26(1) \mathrm{fm} \cdot 3$ and studies of the $\mu=0$ meson spectrum yield $M_{\pi} a=0.79(1)$ and $M_{\pi} / M_{\rho}=0.80(1)$ [6]. For the most part the diquark source $j a=0.04$, though for a few values of $\mu$ the series $j a=0.06,0.04,0.02$ was studied in order to permit a $j \rightarrow 0$ extrapolation. The values of $\mu$ studied are given in Table 1. Configurations are separated by 4 HMC trajectories of typical mean length 0.5 , and are not in general independent.

\footnotetext{
${ }^{3}$ This value is based on string tension measurements on a $12^{3} \times 24$ lattice and supplants those reported in [1, 6].
} 


\begin{tabular}{|ccc|ccc|}
\hline$\mu a$ & $j a$ & No. of Configurations & $\mu a$ & $j a$ & No. of Configurations \\
\hline 0.00 & 0.04 & 210 & 0.50 & 0.02 & 133 \\
0.10 & 0.04 & 200 & 0.50 & 0.04 & 248 \\
0.25 & 0.04 & 117 & 0.50 & 0.06 & 76 \\
0.30 & 0.02 & 78 & 0.55 & 0.04 & 410 \\
0.30 & 0.04 & 133 & 0.60 & 0.04 & 441 \\
0.30 & 0.06 & 70 & 0.70 & 0.02 & 178 \\
0.35 & 0.04 & 124 & 0.70 & 0.04 & 382 \\
0.40 & 0.04 & 175 & 0.70 & 0.06 & 70 \\
0.45 & 0.04 & 281 & 0.90 & 0.04 & 65 \\
\hline
\end{tabular}

Table 1: Number of configuations examined at each value of chemical potential.

Quark propagators on each configuration were calculated by all-to-all techniques [7] using time, spin and flavour dilution. Whilst this equates to constructing $16 \times 4 \times 2=128$ dilution vectors per propagator and thus performing 256 inversions per configuration, at $\mu \neq 0$ this was required for acceptable statistical precision. These were saved to disk and stitched into correlation functions according to the formulæ (7), (99). (11), (12) and (14). Disconnected diagrams relevant for each state were calculated and saved seperately; with the current level of statistics these contributions are both noisy and compatable with zero and so only the connected pieces are presented in this initial study.

To construct kaon correlators, partial quenching was used for the strange propagators, i.e. a further set of propagators was calculated on a $\mu \neq 0$ gauge configuration using $\mathcal{M}(\mu=0 ; j=0.04)$. This choice of $j$ ensures degeneracy of eg. $K$ and pion states at $\mu=0$. The two different sets of propagators were then stitched together with the relevant operators to form $K$ and $K^{*}$ states. We reiterate that the $s$ quark mass used was identical to that of the $u / d$, and clearly whilst the kaon states contain contributions from virtual $u / d$ loops, there is no $s$-quark sea contribution at this stage.

To extract masses the meson correlation functions were fitted to a cosh function. The time range was adjusted to achieve a stable fit while minimising the obtained $\chi^{2} / N_{\mathrm{df}}$. For $\mu \neq 0$ states with baryon number $B \neq 0$ such as the diquarks and the kaons are no longer degenerate with their anti-particles. This results in correlators which are no longer time-symmetric and must be fitted by a sum of two independent exponentials. This can lead to problems with fitting as a 4 parameter fit is more susceptible to noise than one with just 2. Naïvely in the vacuum phase below onset the states' masses receive an additive contribution $\pm \mu B N_{c}$, and so as $\mu$ increases the correlation function is increasingly dominated by the lighter of the particle-antiparticle pair, which is the one with $B<0$. For $\mu>0$ the heavier state rapidly becomes very difficult to fit. 


\section{Results}

All results presented here come purely from analysis of hadron correlators formed from connected quark propagators. The result for the pion mass at $\mu=0$ is $M_{\pi} a=0.800(3)$. Chiral perturbation theory $(\chi \mathrm{PT})$ [8], which models the weakly-interacting dilute Bose gas forming at onset, can be used to predict the onset chemical potential $\mu_{o}$ where the transition from vacuum to the superfluid phase occurs. This prediction, which strictly speaking only applies when there is a separation of scales between the pion and heavier hadrons, is that $\mu_{o}=M_{\pi} / 2$. In our case, this suggests that the onset in the $j \rightarrow 0$ limit occurs at $\mu_{o} a \simeq 0.4$. As $\mu$ is increased we found that more iterations are required to perform an inversion of the quark matrix [1], especially once $\mu>\mu_{o}$ due to the large density of small eigenvalues of $\mathcal{M}$ in the neighbourhood of the origin in this regime.

In principle all our results should be extrapolated to the "physical" limit $j \rightarrow 0$, but unfortunately available resources preclude a systematic study for all $\mu$. Here we follow [1] by studying $j a=0.02, \ldots, 0.06$ at three representative $\mu$ points: just below onset, just above onset, and well into the superfluid phase. The results for $M_{\pi}$ and $M_{\rho}$ are shown in Fig. 1, Within the limits of statistical precision a linear extrapolation $j \rightarrow 0$ is valid. It is notable that the pi and rho masses have opposite slopes as functions of $j$, and that the slopes change sign across the onset transition between $\mu a=0.3$ and $\mu a=0.5$.
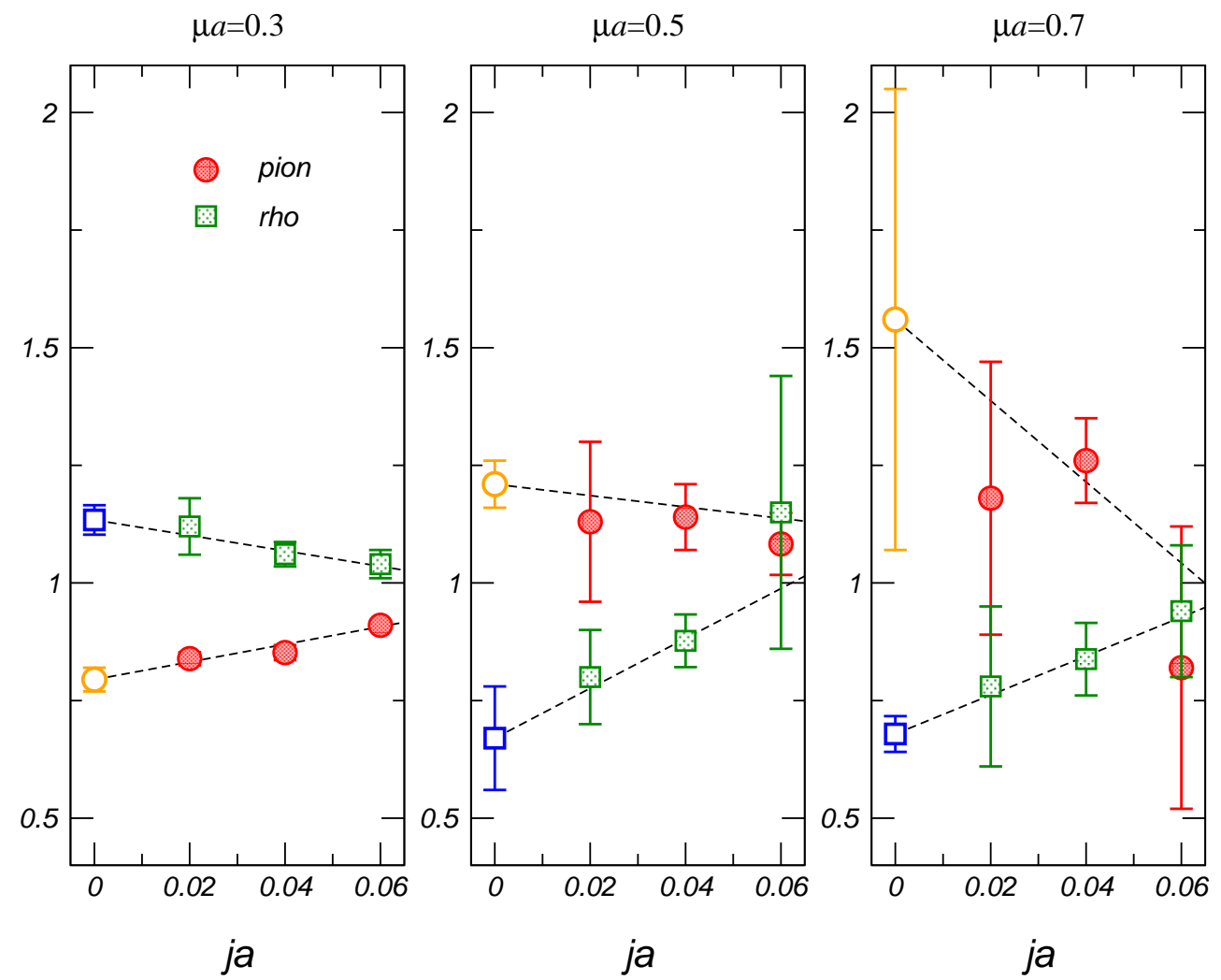

Figure 1: Pi and rho masses as a function of diquark source strength $j$ for three representative values of $\mu$, together with their values extrapolated to $j=0$. 
Results for the meson spectrum as a function of $\mu$ are displayed in Fig. 2. At $\mu=0$

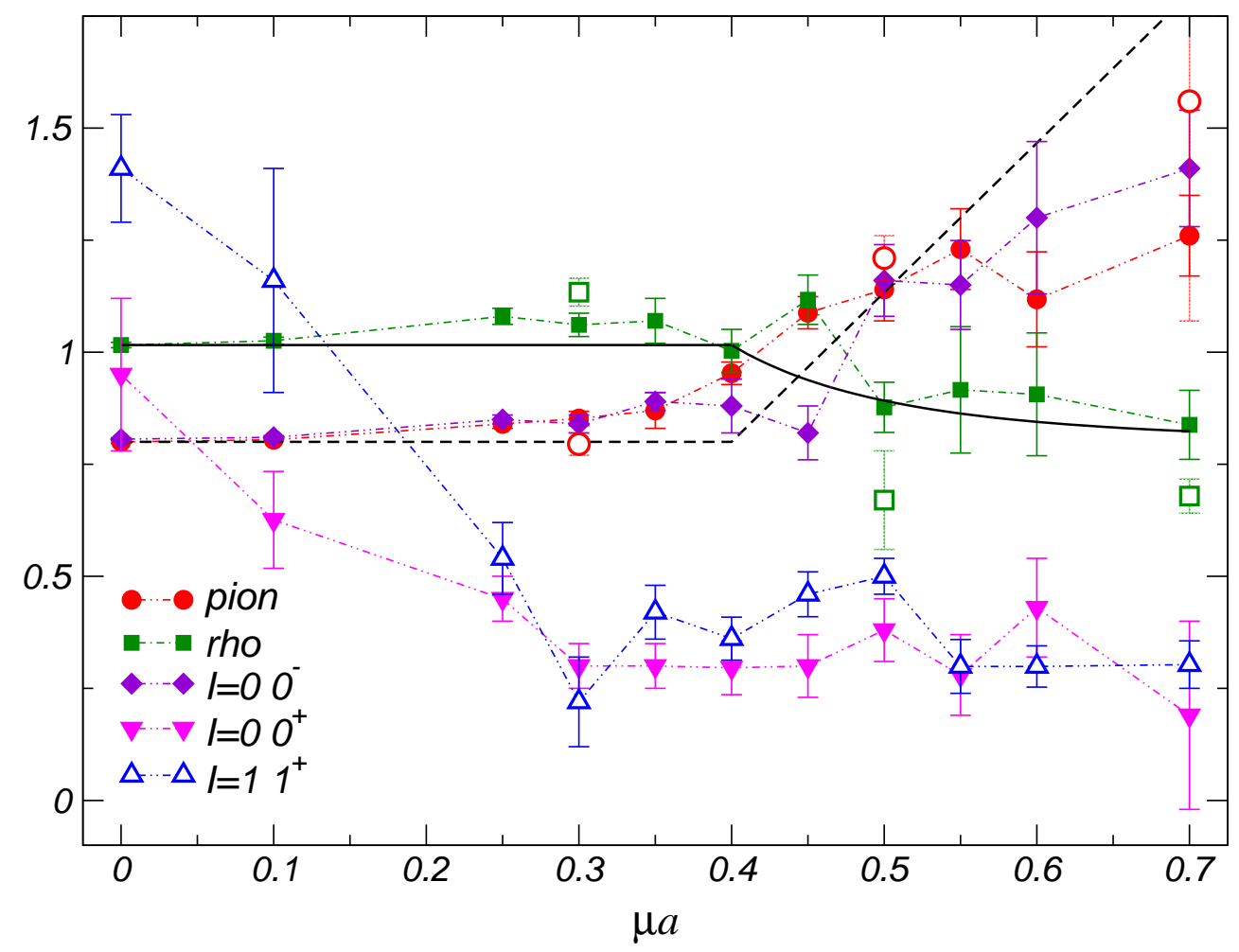

Figure 2: Meson masses as a function of $\mu$ for $j a=0.04$. The dotted line is the $\chi \mathrm{PT}$ prediction for the pion and the solid line is the prediction for the rho from [11. Open symbols denote extrapolations to $j \rightarrow 0$.

the isovector $0^{-}$and $1^{-}$states, ie. the pion and rho, are consistent with the values found in [1, 6]. Throughout the vacuum phase (i.e. $\left.\mu<\mu_{o}\right) M_{\pi}$ and $M_{\rho}$ are more or less constant as expected for states with $B=0$, although they do increase slightly but significantly for $\mu a>0.2$. For the pion this appears to be a $j \neq 0$ artifact vanishing in the $j \rightarrow 0$ limit, but things are not so clear for the noisier rho. At and beyond onset at $\mu_{o} a \simeq 0.4$ the pion and rho signals become much noiser as reflected in the error bars, but it is still possible to identify trends. The pion starts to become heavier at onset and appears to increase in mass monotonically with $\mu$ in the limit $j \rightarrow 0$. The $j=0$ $\chi \mathrm{PT}$ prediction $M_{\pi}=M_{\pi}(\mu=0) \theta\left(\mu_{0}-\mu\right)+2 \mu \theta\left(\mu-\mu_{o}\right)$ [8] (dotted line) is followed in a qualitative sense. The increase of the pion mass post-onset is characteristic of a state formed from $q$ and $\bar{q}$ with a symmetric combination of quantum numbers under the residual global symmetries (i.e. the $P_{S}$ state in the notation of [8]) in a theory with Dyson index $\beta_{D}=1$. The same trend is seen in $\mathrm{QC}_{2} \mathrm{D}$ simulations with staggered fermions in the adjoint representation [9], but the opposite, namely a decrease in $M_{\pi}$ post-onset, is found in a theory with $\beta_{D}=4$ such as $\mathrm{QC}_{2} \mathrm{D}$ with fundamental staggered quarks [4].

By contrast post-onset the rho becomes significantly lighter, in agreement with the 
result found in simulations on $4^{3} \times 8$ with significantly heavier quarks by the Hiroshima group [3]. This effect becomes stronger as $j \rightarrow 0$. Reduction of $M_{\rho}$ in a nuclear medium has been proposed to explain the low mass lepton pair enhancement observed in heavy ion collisions [10]. With the exception of the point at $\mu a=0.45 M_{\rho}$ seems to follow the predictions of an effective model description of spin-1 excitations in $\mathrm{QC}_{2} \mathrm{D}$ [11], shown as a solid line in Fig. 2, Our data suggest, however, that the transition is sharper than that predicted by the model.

Results for isovector $0^{+}$and isoscalar $1^{+}$states are omitted from Fig. 2 because the data are too noisy to fit, and for the isoscalar $1^{-}$for the sake of clarity. The latter follows the rho almost exactly until $\mu a>0.5$ at which point it becomes too noisy to measure. We do, however, include results for the isoscalar $0^{-}$. Since only the connected diagrams are currently being considered the difference between these two states and the pion and rho is just the sign of the anomalous term in (17],9), The fact that they are the same until onset shows that this term is negligible in this regime. Beyond onset, there is a small window in which the $0^{-}$in the isoscalar channel is significantly lighter than the isovector, and is indeed roughly degenerate with the $I=00^{-}$diquark of Fig. 4, to be discussed below.

The two remaining mesons shown in Fig. 2, the isovector $1^{+}$and isoscalar $0^{+}$, show a similar behaviour, both starting off relatively heavy (and noisy) and then rapidly dropping as $\mu$ increases. By $\mu a=0.3$ they have reached a minimum and stay more or less constant as $\mu$ increases further. We shall argue below that the low mass of the $0^{+}$state is due to its overlap with the Goldstone boson in the superfluid phase, when baryon number ceases to be a good quantum number; the low mass of the $1^{+}$is more unexpected.

To understand the physics of the diquark sector, it is helpful to begin with the Higgs and Goldstone states of Eq. (14) with a varying diquark source strength $j$. Fig. 3 shows Higgs and Goldstone masses as functions of $\mu$ at $j a=0.04$. The insets show how the two states scale with $j$ at three selected values of $\mu$. Below onset Higgs and Goldstone are degenerate, both scaling approximately linearly 4 with $j$. Post onset the degeneracy is broken, and the relation $M_{\text {Gold }} \propto \sqrt{j}$ predicted in $\chi \mathrm{PT}\left[8\right.$ ] appears to hold, the $\chi^{2} / N_{\mathrm{df}}$ being $0.67,0.62$ and 1.65 for the 3 linear Higgs extrapolations, and 1.08, 2.69 and 0.24 for the 3 Goldstone extrapolations, which have the form $M_{\text {Gold }}=a \sqrt{ } j+b$ above onset.

Extrapolations to $j=0$ using these scaling assumptions are also displayed on the main graph (triangular symbols). The two states remain degenerate until onset at which point the Goldstone becomes lighter than the Higgs, and appears to become massless as $j \rightarrow 0$. This is a clear manifestation of spontaneous breaking of $\mathrm{U}(1)_{B}$ symmetry breaking for $\mu>\mu_{o}$, implying a superfluid ground state in which baryon number is no longer a good quantum number, and therefore meson and diquark states in principle indistinguishable.

The diquark spectrum in the remaining spin-0 and spin-1 channels is shown in Fig. 4,

\footnotetext{
${ }^{4}$ The pre-onset behaviour $M(j)=M(0)\left(1+b j^{2}\right)^{\frac{1}{4}}$ predicted by $\chi \mathrm{PT}[8$ may be difficult to distingush from linear behaviour in this regime.
} 


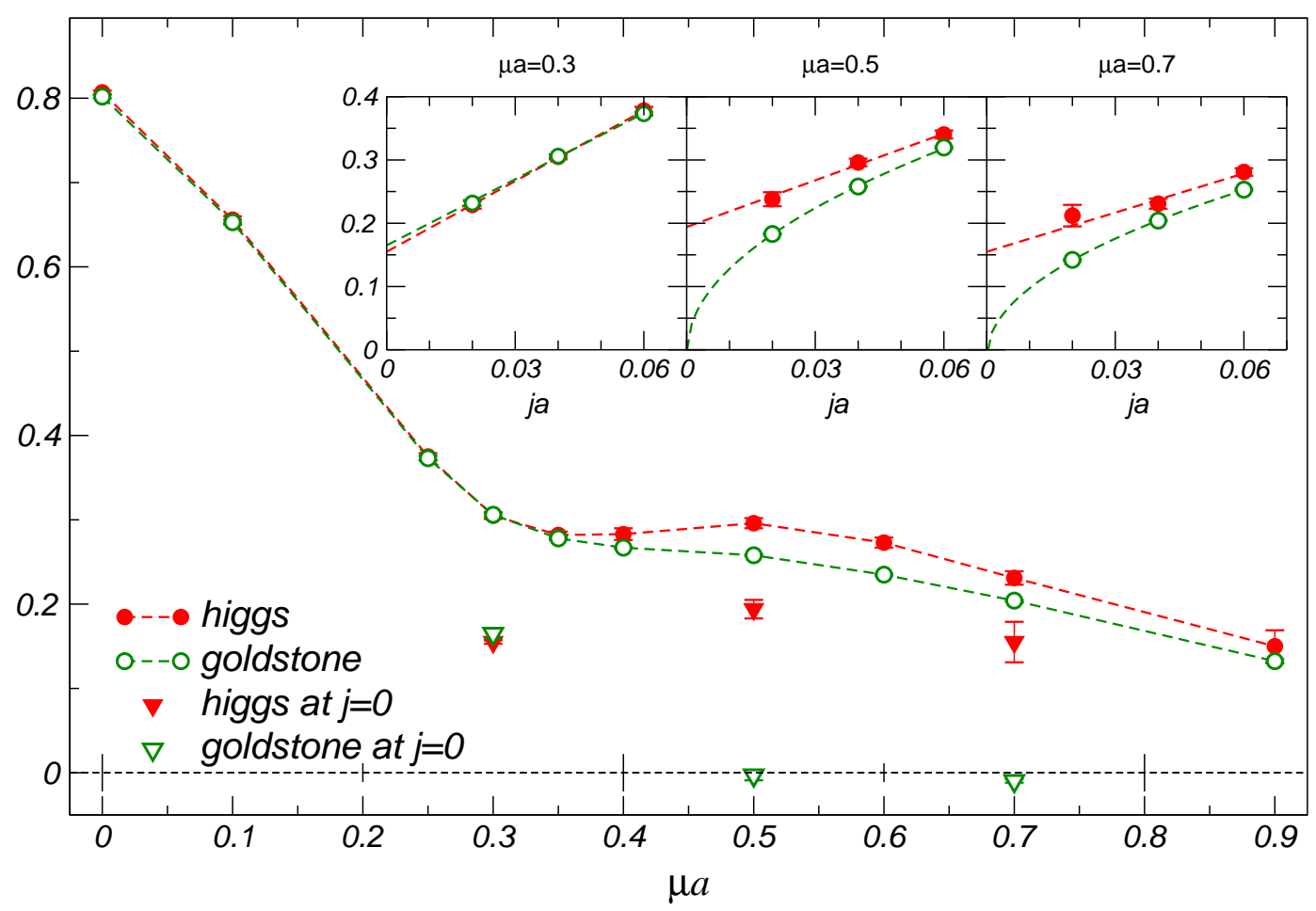

Figure 3: Higgs and Goldstone masses as a function of $\mu$. The two insets show results as $j$ is varied at fixed $\mu a=0.3,0.5,0.7$. Extrapolations to $j=0$ are displayed on the main graph with triangle symbols.

It is striking that the signal-noise ratio is much higher for some diquarks than for the mesons, also seen in simulations with staggered fermions [4]. The two cleanest signals are for the isoscalar $0^{+}$and the isovector $1^{+}$. The first observation is that as a consequence of the symmetry (3) there is a relation between the meson and diquark spectra which holds for $\mu=j=0$ if disconnected diagrams are neglected:

$$
M_{D}\left(J^{P}\right)=M_{M}\left(J^{-P}\right) .
$$

For $0<\mu<\mu_{o}$, during which the physical ground state remains the vacuum, we thus predict $M_{D}\left(0^{+}\right)=M_{\pi} \pm 2 \mu, M_{D}\left(1^{+}\right)=M_{\rho} \pm 2 \mu$, shown as dot-dashed lines in Fig. 4. Indeed both diquark particle-antiparticle pairs behave as expected up to $\mu a \approx 0.3$. Diquark masses are not shown beyond $\mu a=0.25$ as they become unfittable, as explained in Sec. 3. After this both $0^{+}$and $1^{+}$anti-diquark states flatten off and slowly decrease with $\mu$. The other two isoscalar diquarks constructed from local operators, namely the $0^{-}$and $1^{-}$, are extremely heavy and hard to fit below onset, but above onset have a sufficiently good signal for us to deduce masses comparable with $M_{\pi}(\mu=0), M_{\rho}(\mu=0)$. Although the noise in the meson sector is admittedly large, the approximate degeneracy between meson and baryon sectors in the $0^{+}$and $1^{+}$channels seen in Figs. 2 and 4 is consistent with the meson-baryon degeneracy in the superfluid state discussed above. Meson-diquark degeneracy has also been observed in quenched studies at $\mu \neq 0$ with staggered fermions [12]. 


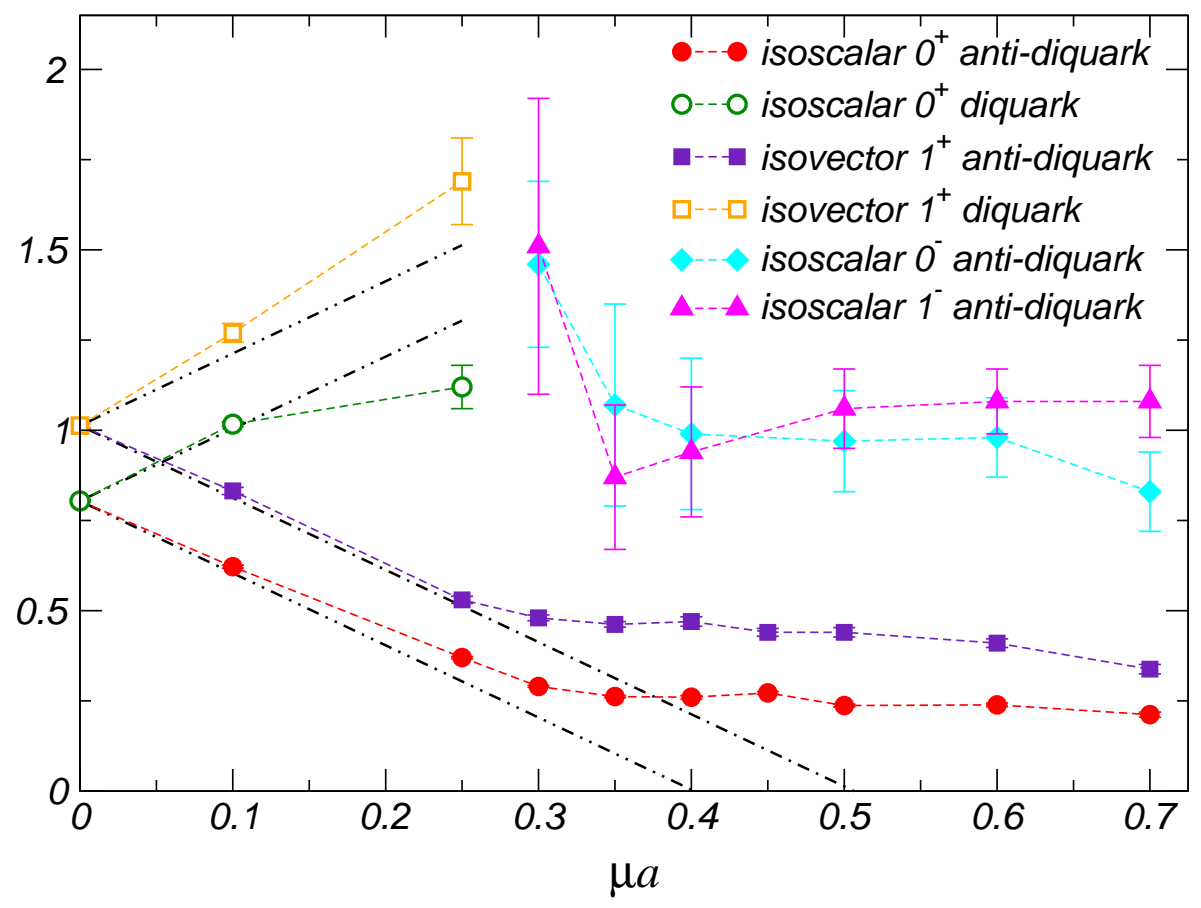

Figure 4: Diquark masses as a function of $\mu$. Only states corresponding to operators with $B<0$ are displayed for $\mu a>0.25$. The dot-dashed lines have intercepts at $M_{\pi, \rho}$, and gradients \pm 2 .

The isoscalar $0^{-}$diquark is a particularly interesting state in $\mathrm{QC}_{2} \mathrm{D}$, since because of meson-baryon mixing in the superfluid phase it has the same quantum numbers as the $\eta^{\prime}$ meson [2], and hence its mass acts as a probe of instanton effects and/or possible restoration of $\mathrm{U}(1)_{A}$ symmetry in a baryonic medium. Unfortunately the current simulations are not close enough to the chiral limit to settle this issue via observation of a $\pi(\mu=0)-\eta^{\prime}(\mu)$ mass splitting.

The kaon spectrum is shown in Fig. 5; since $\mu_{s}$ is assumed to be zero these states in effect carry a baryon charge conjugate to $\mu$, and hence in general their correlators are not forwards-backwards symmetric. Some care must be taken in assigning physical quantum numbers to the states [5]. Consider say the $0^{-}$excitation in a simple model in which the quarks are non-interacting but have mass $m$. Below onset the "light" quark spectrum is split into two branches, a hole branch with energy $E_{h}=\sqrt{p^{2}+m^{2}}-\mu$ and a particle branch with $E_{p}=\sqrt{p^{2}+m^{2}}+\mu$, while the $s$-quark has energy $E_{s}=\sqrt{p^{2}+m^{2}}$ independent of $\mu$. We thus identify a zero momentum " $K^{-}$" state with energy $E_{s}+E_{h}=$ $2 m-\mu$, and a " $K^{+}$" with $E_{s}+E_{p}=2 m+\mu$. Above onset, the particle branch becomes filled up to some momentum $p_{F}=\sqrt{\mu^{2}-m^{2}}$, so that the light quark hole state now costs energy $E_{h}=\mu-\sqrt{p^{2}+m^{2}}$. The $K^{-}$state now has $E=E_{s}+E_{h}=\mu$ independent of $\vec{p}$, implying a very small in-medium velocity. If the discussion is modified to include the effect of a non-zero diquark source, then the behaviour both below and above onset 


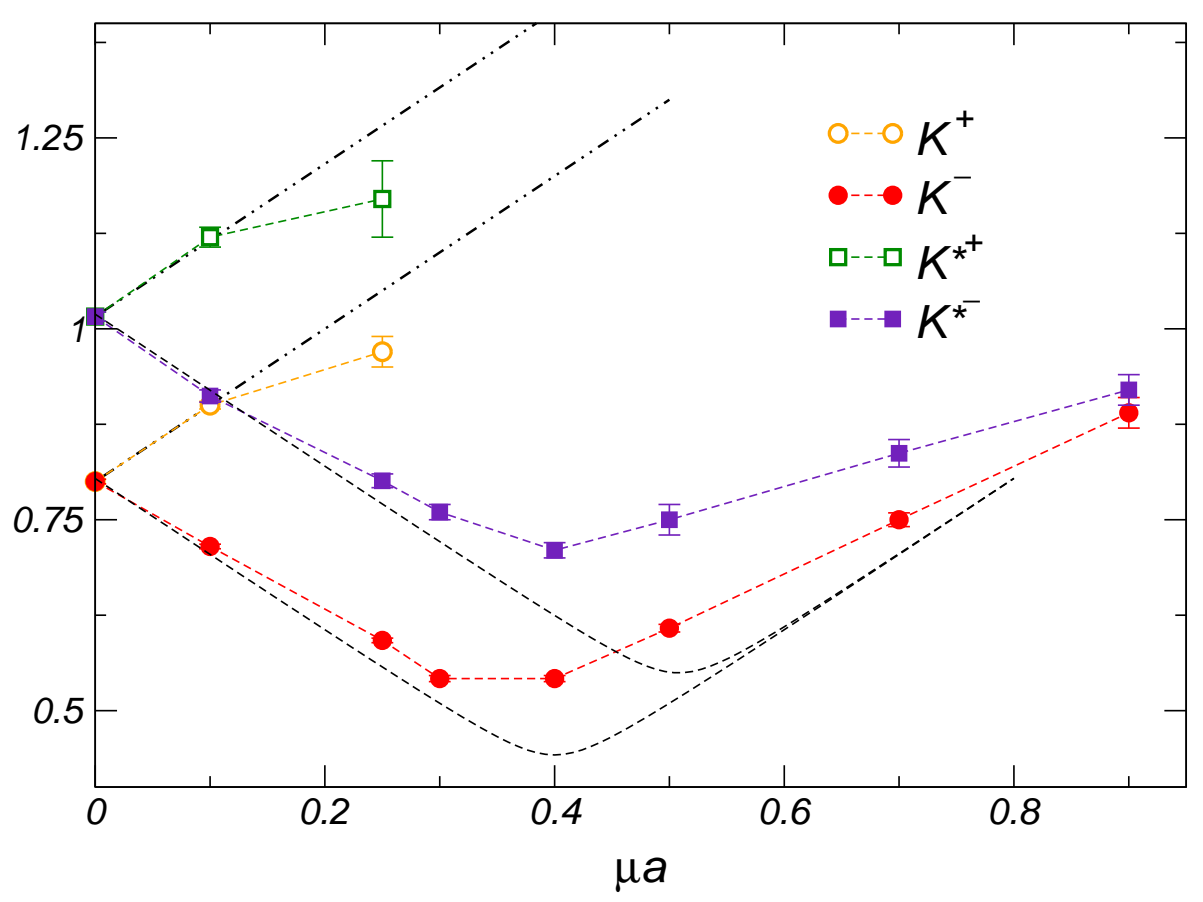

Figure 5: Kaon spectrum as a function of $\mu$. Only the $K^{-}$states are displayed for $\mu a>0.25$. Dotdashed lines correspond to $M_{K}=M_{\pi, \rho}(\mu=0)+\mu$, while the dashed line corresponds to (16) with $\vec{p}=\overrightarrow{0}$ and $m=M_{\pi, \rho}(\mu=0) / 2$

is described by the single solution

$$
E(\vec{p})=\sqrt{\left(\mu-\sqrt{p^{2}+m^{2}}\right)^{2}+j^{2}}+\sqrt{p^{2}+m^{2}+j^{2}} .
$$

Fig. 5 shows that this simple picture works qualitatively quite well in both $K$ and $K^{*}$ channels, even once interactions are present, if we replace the vacuum mass $2 m$ with the $\mu=0$ mass of the corresponding meson from Fig. 2. The observed $M_{K}$ always exceeds the model prediction, but appears to match both at $\mu=0$ and $\mu \rightarrow \infty$ and retains a minimum for $\mu \approx \mu_{o}$. There is thus a significant range $\mu_{o}<\mu \lesssim M_{K}$ in which $M_{K^{-}}$ lies below its vacuum value, and similarly for $M_{K^{*-}}$. This offers theoretical support for the idea that in-medium modification of kaon masses in nuclear matter leads to deeply-bound $K^{-}$-nuclear states [13], for which there is some experimental support from scattering kaons on light nuclei [14]. Of course, simulations with realistic quark masses are needed before this observation can be taken seriously, not to mention the extension from two colours to three. Nonetheless, we can identify a minimal requirement for the existence of kaonic-nuclear bound states, namely $M_{K}>\mu_{o}$. This inequality is satisfied in QCD. 


\begin{tabular}{|cc|cc|cc|}
\hline Quantum Numbers & & \multicolumn{2}{|c|}{ Meson } & \multicolumn{2}{c|}{ Diquark } \\
& & $\mu<\mu_{o}$ & $\mu>\mu_{o}$ & $\mu<\mu_{o}$ & $\mu>\mu_{o}$ \\
\hline$I=0$ & $0^{+}$ & noisy & good & good & good \\
$I=0$ & $0^{-}$ & good & noisy & noisy & good \\
$I=0$ & $1^{-}$ & good & noisy & noisy & good \\
$I=0$ & $1^{+}$ & no signal & no signal & no state \\
\hline$I=1$ & $0^{+}$ & no signal & no signal & \multicolumn{2}{|c|}{ no state } \\
$I=1$ & $0^{-}$ & good & noisy & \multicolumn{2}{|c}{ no state } \\
$I=1$ & $1^{-}$ & good & noisy & no state \\
$I=1$ & $1^{+}$ & noisy & good & good & good \\
\hline
\end{tabular}

Table 2: Informal summary of the success of our hadron fits.

\section{Discussion}

It is helpful to summarise our findings in the form of a table in which the quality of our fits both below and above onset is given. The main achievements of this study have been observations of:

- The reversal of the pion and rho levels on crossing from vacuum into a baryonic medium. In the vacuum $\mu<\mu_{o} M_{\pi, \rho}$ is approximately constant, probably because there is no diquark state with the same quantum numbers with which to mix.

- The breaking of the degeneracy between Higgs and Goldstone diquark states for $\mu>\mu_{o}$, the Goldstone mass scaling as $\sqrt{j}$ in accordance with general theoretical properties of spontaneous symmetry breaking by condensation of fermion pairs.

- Further evidence for meson-baryon mixing in the degeneracy of $I=00^{+}$and $I=1$ $1^{+}$states for $\mu>\mu_{o}$. Post onset the $1^{+}$appears to be the next lightest state after the Goldstone and Higgs. The fact that the mesons with these quantum numbers appear not to have constant mass even pre-onset (see Fig. 2) can also be ascribed to meson-baryon mixing, since for $j \neq 0$ there is a non-zero amplitude for $\bar{\psi} \psi$ to project onto a baryon.

- Evidence of the possibility of bound kaons in nuclear matter.

Our study has uncovered little about the effects of a second deconfining phase transition suspected to occur at $\mu_{d} a \approx 0.65$ on this system [1]. The only possible discernable trend is a levelling off of the already massive pion state for $\mu a \gtrsim 0.5$ seen in Fig. 2. Statistical noise increasing with $\mu$, however, makes this observation provisional at best. In a deconfined phase we might expect mesons and baryons to be formed from particle-hole and particle-particle pairs in the neighbourhood of a Fermi surface, and it is possible that the local operators used in this study have a poor projection onto the true quasiparticle excitations. We hope that studies of meson and diquark wavefunctions currently 
in progress, as well as extended operators for the $I=00^{+}$and $I=11^{+}$mesons and enhanced statistics for the disconnected diagrams, will clarify the situation.

\section{Acknowledgements}

JIS has been supported by IRCSET award SC/03/393Y and SFI grant 04/BRG/P0266. The project was enabled with the assistance of IBM Deep Computing. We'd also like to thank Justin Foley for helpful discussions.

\section{References}

[1] S.J. Hands, S. Kim, and J.I. Skullerud, Eur. Phys. J. C48 (2006) 193.

[2] T. Schäfer, Phys. Rev. D 65 (2002) 094033.

[3] S. Muroya, A. Nakamura and C. Nonaka, Phys. Lett. B551 (2003) 305.

[4] J.B. Kogut, D.K. Sinclair, S.J. Hands and S.E. Morrison, Phys. Rev. D 64 (2001) 094505.

[5] S.J. Hands, B. Lucini and S.E. Morrison, Phys. Rev. D65 (2002) 036004.

[6] S.J. Hands, S. Kim and J.I. Skullerud, Eur. Phys. J. A31 (2007) 787.

[7] J. Foley et al., Comput. Phys. Commun. 172 (2005) 145.

[8] J.B. Kogut, M.A. Stephanov, D. Toublan, J.J.M. Verbaarschot, and A. Zhitnitsky, Nucl. Phys. B582 (2000) 477.

[9] S.J. Hands, I. Montvay, S.E. Morrison, M. Oevers, L. Scorzato and J.I. Skullerud, Eur. Phys. J. C17 (2000) 285.

[10] B. Lenkeit et al. [CERES-Collaboration], Nucl. Phys. A661 (1999) 23c.

[11] J.T. Lenaghan, F. Sannino, and K. Splittorff, Phys. Rev. D65, 054002 (2002).

[12] P. Giudice and S.J. Hands, arXiv:hep-lat/0703001.

[13] Y. Akaishi and T. Yamazaki, Phys. Rev. C65 (2002) 044005.

[14] T. Suzuki et al., Phys. Lett. B597 (2004) 263;

M. Agnello et al. [FINUDA Collaboration], Phys. Rev. Lett. 94 (2005) 212303;

T. Kishimoto et al., Nucl. Phys. A754 (2005) 383. 